\title{
Recubrimiento controlado de sustratos de vidrio con nanobastones metálicos
}

\section{Controlled coverage of glass substrates with metallic nanorods}

\author{
Marina Verónica Gutiérrez ${ }^{1}$, María Yanela Paredes ${ }^{1}$, \\ Alberto Franco Scarpettini ${ }^{1,2}$
}

\author{
${ }^{1}$ Laboratorio de Optoelectrónica y Metrología Aplicada, Facultad Regional Delta, Universidad Tecnológica Nacional, \\ San Martín 1171, Campana, Argentina \\ ${ }^{2}$ Consejo Nacional de Investigaciones Científicas y Técnicas, Argentina \\ e-mail: ascarpettini@frd.utn.edu.ar
}

\begin{abstract}
RESUMEN
Se realizó la síntesis de nanobastones de oro monodispersos con una eficiencia superior al $80 \%$ sobre el total de nanopartículas, caracterizado por una resonancia plasmónica longitudinal cercana a los $800 \mathrm{~nm}$. Se modificaron superficialmente sustratos de vidrio y se los recubrió con los nanobastones sintetizados, con control de la densidad superficial. Se monitoreó la dinámica del recubrimiento a través de espectros de extinción, y se observó una densidad máxima de saturación dada por repulsión electrostática y un tiempo característico del proceso. Luego de alcanzada la saturación de nanobastones por unidad de área se observa un ensanchamiento de las resonancias hacia el infrarrojo, debido a interacciones entre los nanobastones por producirse agregaciones sobre la superficie. Estos resultados tienen aplicación inmediata en el diseño y fabricación de dispositivos plasmónicos, por ejemplo en el sensado molecular.
\end{abstract}

Palabras clave: Nanobastones de oro, Plasmónica, Sustratos nanoestructurados.

\section{ABSTRACT}

Monodispersed gold nanorods were synthesized with efficiency above $80 \%$ of all nanoparticles, characterized by longitudinal plasmon resonance around $800 \mathrm{~nm}$. Glass substrates were surface modified and covered with the synthesized nanorods with control of surface density. Coverage dynamics was monitored through extinction spectra, and a saturation maximum density was observed, given by electrostatic repulsion, and a characteristic time of the process. A widening of the resonance towards the infrared is observed after reaching the saturation of nanorods per unit area, due to interactions between nanorods on account of surface aggregation. These results have immediate application in the design and manufacture of plasmonic devices, for example in molecular sensing.

Keywords: Gold nanorods, Plasmonics, Nanostructured substrates.

\section{INTRODUCCIÓN}

Uno de los objetivos de la plasmónica es el control preciso de las propiedades ópticas de la materia nanoestructurada con el fin de lograr, por ejemplo, sensores eficientes y sensibles [1, 2$]$, fuentes de luz ultra com-

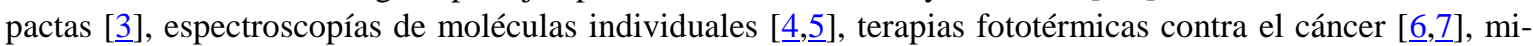
croscopías ópticas de resolución nanométrica [ㅇ,9], celdas solares [10], entre otras aplicaciones. Para lograr esto es indispensable el diseño a medida de nanoestructuras, su fabricación con alta eficiencia y repetitividad, $\mathrm{y}$ el control preciso de sus formas y dimensiones.

En esta línea, los nanobastones de oro (NBOs) son atractivos por su simplicidad y porque poseen resonancias plasmónicas superficiales en el rango visible e infrarrojo del espectro electromagnético. La longitud de onda de estas resonancias depende, entre otros factores, de las dimensiones de los NBOs. Si se tiene control sobre su relación de aspecto se puede sintonizar una resonancia relativamente angosta [11]. De este modo es posible construir un dispositivo plasmónico resonante a una longitud de onda deseada, sintetizando NBOs de dimensiones adecuadas y fabricando una nanoestructura usando los NBOs como ladrillos primordiales.

Se han reportado varios ejemplos de sensado molecular usando NBOs en una solución coloidal, pero 
para lograr resultados precisos y confiables se requiere ubicarlos en la superficie de un sustrato determinado. Es importante obtener control sobre el grado de recubrimiento de un sustrato con nanopartículas y sobre cómo se agregan en dímeros, trímeros o grupos mayores porque las resonancias plasmónicas, y por consiguiente sus propiedades ópticas, dependen fuertemente del número de nanopartículas presentes y de las interacciones entre ellas $[\underline{12}, \underline{13}]$. Una de las formas de realizar estos recubrimientos es a través de la evaporación del solvente, pero este método no permite recubrir homogéneamente el sustrato y es muy difícil llevar a cabo recubrimientos en áreas extensas $[\underline{14}, \underline{15}]$. Otros métodos se basan en la interacción electrostática para fijar al sustrato los NBOs, pues la capa de surfactante que los rodea les confiere una carga positiva. Algunos autores proponen recubrir un sustrato de vidrio con capas moleculares de manera tal de tener una carga externa neta negativa, y al sumergirlo en el coloide, los NBOs colisionan y se adsorben hasta que la carga superficial total impide que otros NBOs superen el potencial electrostático [16,17]. Una variante es evaporar oro sobre el sustrato y pegar una monocapa de tioles, generando así una capa externa negativa [18]. Otros autores proponen recubrir los NBOs con un polímero negativo para cambiarles la carga, y utilizar un sustrato con moléculas de carga positiva [19-23]. Una variante es reemplazar el surfactante por otro que sea negativo, y utilizar nuevamente un sustrato con moléculas positivas [24].

En este trabajo realizamos una inmersión del sustrato en la solución coloidal de NBOs para posibilitar recubrimientos extensos, uniformes y de densidad predecible, aumentando la capacidad de detección y reconocimiento molecular del sensor. La fuerza iónica y la concentración del surfactante son factores importantes en la dinámica de recubrimiento. Una concentración muy alta de surfactante apantalla la carga superficial del sustrato, además de generar cristalizaciones orgánicas indeseadas. Una concentración muy baja puede ocasionar inestabilidades en el coloide. La dinámica de las interacciones entre NBOs y NBO-sustrato guía el proceso de recubrimiento y ensamblado superficial, y pequeñas variaciones de la fuerza iónica y de la concentración del surfactante pueden ocasionar resultados muy diversos [17].

Varios autores reportan la síntesis química de NBOs monodispersos con muy buena eficiencia [25-27]. En esta última década se han documentado avances importantes en el crecimiento mediado por semillas de NBOs en medio acuoso, con control sobre el tamaño y su uniformidad, y la sintonización espectral de la resonancia plasmónica. La idea básica de este proceso es usar micelas de bromuro de hexadeciltrimetilamonio (CTAB), que es un surfactante catiónico, como moldes blandos para dirigir el crecimiento de los NBOs. Algunos autores reportan que la unión preferencial de las moléculas de CTAB sobre determinadas caras cristalinas incide en la formación de los NBOs. Se ha demostrado que la presencia de pequeñas cantidades de iones plata en la solución de crecimiento interviene también en la formación de NBOs y en el control preciso de sus dimensiones [25, 26]. En general, las moléculas de surfactante en solución acuosa se autoensamblan formando micelas, y se puede lograr una mejora en esta micelización y por consiguiente en la monodispersión de tamaño y forma agregando un compuesto aromático al surfactante [27].

En el presente trabajo sintetizamos NBOs coloidales monodispersos y luego recubrimos sustratos de vidrio con ellos, usando grupos aminosilano y poliestireno para su funcionalización y fijación. Estudiamos la dinámica de recubrimiento a través de espectros de extinción. Mostramos que el tiempo característico de recubrimiento es entre 5 y 6 horas. Una vez alcanzado el recubrimiento máximo se observa un ensanchamiento del plasmón correspondiente a la resonancia longitudinal, debido a interacciones entre los NBOs, posiblemente por movilidad sobre la superficie del sustrato formando grupos de dos o más nanopartículas.

\section{MÉTODOS}

\subsection{Materiales e instrumentación}

Los siguientes productos químicos fueron utilizados sin modificaciones. El tetracloroaurato de hidrógeno trihidratado $\left(\mathrm{HAuCl}_{4} \cdot 3 \mathrm{H}_{2} \mathrm{O},>99,9 \%\right)$, bromuro de hexadeciltrimetilamonio (CTAB, > 98\%), borohidruro de sodio $\left(\mathrm{NaBH}_{4},>98 \%\right)$, ácido 5-bromosalić́lico (> 90\%), nitrato de plata $\left(\mathrm{AgNO}_{3},>99 \%\right)$, (3-aminopropyl)trimethoxysilane (APTMS, > 97\%) y poly(sodium 4-styrenesulfonate) (PSS, $\mathrm{M}_{\mathrm{w}} \sim 70000$ ) fueron comprados a Sigma Aldrich. El ácido ascórbico (> 99,7\%) fue comprado a Biopack. En todos los casos se utilizó agua ultrapura Milli-Q.

Los espectros de extinción de los coloides y los sustratos de vidrio fueron obtenidos con un espectrofotómetro UV-visible Optizen Pop de Mecasys. Para este propósito se utilizó una cubeta UV-visible con la solución acuosa de NBOs, y mediante un dispositivo sencillo de sujeción se situaron los sustratos recubiertos en el camino del haz del espectrofotómetro. Las líneas de base fueron realizadas con cubetas con agua y sustratos sin recubrir, respectivamente. Las imágenes de los NBOs sobre sustratos fueron obtenidas con un microscopio electrónico de barrido (SEM) Zeiss Supra 40, con valores de tensión entre 3 y 5 kV. 


\subsection{Síntesis de nanobastones de oro}

Para preparar la solución semilla se mezclan $5 \mathrm{ml}$ de $\mathrm{HAuCl}_{4} 0,5 \mathrm{mM}$ con $5 \mathrm{ml}$ de CTAB 0,2 M. Luego 0,6 $\mathrm{ml}$ de $\mathrm{NaBH}_{4} 10 \mathrm{mM}$ preparado en el momento se diluye a $1 \mathrm{ml}$ con agua y se agrega a la solución anterior, mezclando rápidamente durante 2 minutos. La solución pasa de un color amarillo a un color amarronado. Se deja esta solución semilla en reposo a temperatura ambiente durante media hora.

Para preparar la solución de crecimiento se disuelven $900 \mathrm{mg}$ de CTAB y $110 \mathrm{mg}$ de ácido 5bromosalicílico en $25 \mathrm{ml}$ de agua a $60^{\circ} \mathrm{C}$. Se deja enfriar hasta $30^{\circ} \mathrm{C}$ y luego se agrega $1,2 \mathrm{ml} \mathrm{de} \mathrm{AgNO}_{3} 4$ $\mathrm{mM}$. Se deja en reposo durante 15 minutos, y luego se agrega $25 \mathrm{ml} \mathrm{de} \mathrm{HAuCl}_{4} 1 \mathrm{mM}$ mientras se mezcla lentamente durante otros 15 minutos. Se agrega luego $0,2 \mathrm{ml}$ de ácido ascórbico $64 \mathrm{mM}$ y se mezcla rápidamente durante 2 minutos, se observa que la solución se torna incolora.

Finalmente, se agrega $0,08 \mathrm{ml}$ de solución semilla dentro de la solución de crecimiento, mezclando 30 segundos y luego dejando en reposo un día a temperatura ambiente. Luego se purifica mediante centrifugado a 8500 rpm durante 25 minutos, se remueve el sobrenadante y se redispersa en agua. Se repite con 2 centrifugados adicionales a $8000 \mathrm{rpm}$ durante 20 minutos, removiendo el sobrenadante y redispersando en agua. Este procedimiento de purificación elimina el exceso de surfactante en solución sin afectar el recubrimiento micelar de los NBOs. Esto es importante para evitar que se genere una capa iónica sobre el sustrato que apantalle la carga eléctrica y evite la adsorción de nanopartículas.

\subsection{Recubrimiento de sustratos de vidrio}

Los portaobjetos de vidrio son lavados con agua ultrapura y sonicados durante 30 minutos. En una primera etapa son sumergidos en forma vertical en una solución de APTMS diluida 1:100 durante 1 hora en ambiente oscuro. Luego se lavan con abundante agua y se sumergen en una solución de PSS de $20 \mathrm{mg} / \mathrm{ml}$ durante 1 hora. Finalmente son sumergidos en la solución purificada de NBOs a temperatura ambiente sin mezclado y retirados en tiempos distintos para evaluar el grado de recubrimiento y agregación alcanzado.

\section{RESULTADOS Y DISCUSIÓN}

El crecimiento mediado por semillas en presencia del surfactante CTAB, según el protocolo descripto en la sección anterior, dio por resultado NBOs monodispersos. En la Figura 1 se observa el espectro de extinción de la solución coloidal de NBOs sintetizados. Posee un pico correspondiente a la resonancia plasmónica transversal en $520 \mathrm{~nm}$ aproximadamente, y un pico más intenso correspondiente a la resonancia longitudinal cercano a $775 \mathrm{~nm}$. En la imagen SEM de la Figura 2 puede observarse el coloide evaporado sobre un sustrato, con una población mayoritaria de NBOs de tamaño uniforme y medidas aproximadas de $45 \mathrm{~nm}$ de largo por $15 \mathrm{~nm}$ de ancho. Se observa también una pequeña cantidad de nanopartículas de forma esferoide de tamaño variable comprendido entre 10 y $40 \mathrm{~nm}$. Realizando una estadística de conteo sobre una decena de imágenes SEM se obtiene una eficiencia de producción de NBOs por encima del $80 \%$ sobre el total de nanopartículas sintetizadas.

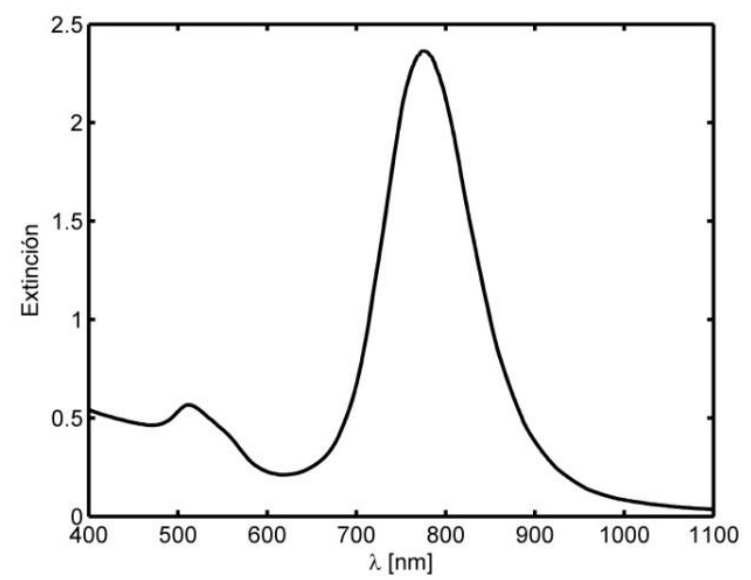

Figura 1: Espectro de extinción de los NBOs sintetizados. Se observan los dos picos característicos correspondientes a las resonancias plasmónicas en el eje transversal del nanobastón (en el verde) y en el eje longitudinal (más intenso, en el rojo-IR). 


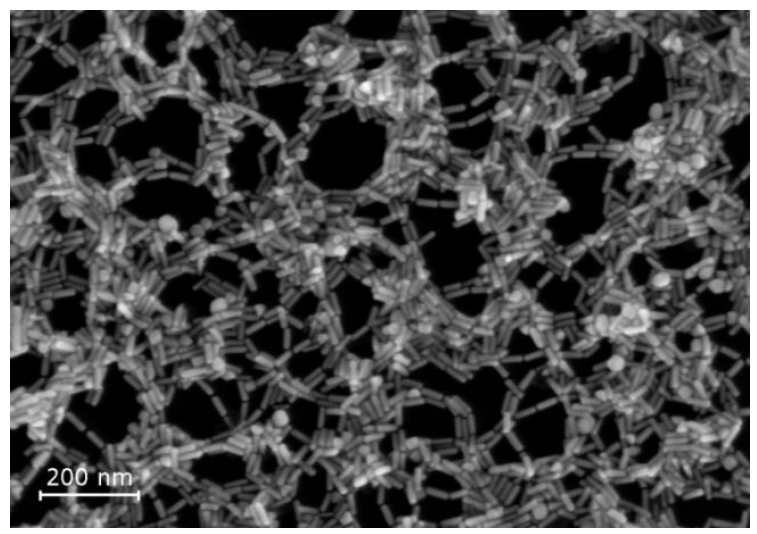

Figura 2: Imagen de microscopía electrónica de barrido (SEM) de los NBOs depositados en un sustrato conductor por evaporación del solvente. Se observa una gran mayoría de NBOs monodispersos en tamaño y forma, y una pequeña cantidad de nanoesferoides.

La estrategia de recubrimiento de sustratos con NBOs es utilizar enlaces electrostáticos entre los NBOs rodeados por el CTAB positivo y la capa de PSS cargada negativamente. Para ello, primero se sumergen portaobjetos previamente lavados y sonicados en una solución diluida de APTMS durante una hora. Este procedimiento genera una monocapa que se enlaza covalentemente al silicio del portaobjetos y expone el grupo amino cargado positivamente hacia el exterior [28]. Luego se lavan con abundante agua para remover las moléculas de APTMS no adsorbidas y se sumergen en una solución de PSS durante una hora. De esta forma las moléculas de PSS se depositan sobre el APTMS y revierten la carga superficial, siendo ahora negativa. Finalmente se vuelven a lavar bien y se sumergen en la solución coloidal de NBOs. Estos coloides fueron purificados mediante 3 centrifugaciones sucesivas para remover el exceso de CTAB, redispersando en agua. La bicapa de surfactante cargada positivamente permite a los NBOs unirse al PSS, cuando colisionan con estas moléculas impulsados por el movimiento browniano.

Los portaobjetos fueron retirados de la solución de NBOs a tiempos distintos para evaluar el grado de recubrimiento a través de espectros de extinción e imágenes de microscopía electrónica. En la Figura 3 se observa la evolución de los espectros para distintos tiempos de inmersión del portaobjeto en el coloide: 1/2 hora, 1 hora, 2 hs, 4 hs, 24 hs y 72 hs. El pico correspondiente al plasmón longitudinal crece con el tiempo, indicando una presencia cada vez mayor de NBOs sobre la superficie del sustrato, y se acerca asintóticamente a un valor de saturación. Este valor límite está dado por la repulsión electrostática entre los NBOs adsorbidos y los NBOs en solución, que frena el proceso de recubrimiento. Esta dinámica de recubrimiento está gobernada por la ecuación:

$$
c(t)=c_{\text {sat }}\left(1-e^{-t / \tau}\right)
$$

que modela la adsorción de coloides sobre sustratos en el tiempo, teniendo en cuenta las diferentes interacciones intervinientes [28]. El parámetro $c_{s a t}$ es el cubrimiento de saturación dado por la repulsión electrostática entre NBOs, y $\tau$ es el tiempo característico de recubrimiento. En la Figura 4 se muestra un gráfico con las amplitudes de los picos del plasmón longitudinal de los espectros de la Figura 3, en función del tiempo de inmersión de los portaobjetos en la solución coloidal de NBOs. Se observa el comportamiento exponencial de la ec. (1), y se agrega el ajuste de los datos con dicha ecuación. El tiempo característico de este proceso es de $(5,5 \pm 2,9)$ horas, y se alcanza una absorbancia de saturación de $(0,29 \pm 0,05)$ correspondiente a un número máximo de NBOs por unidad de área del sustrato. 


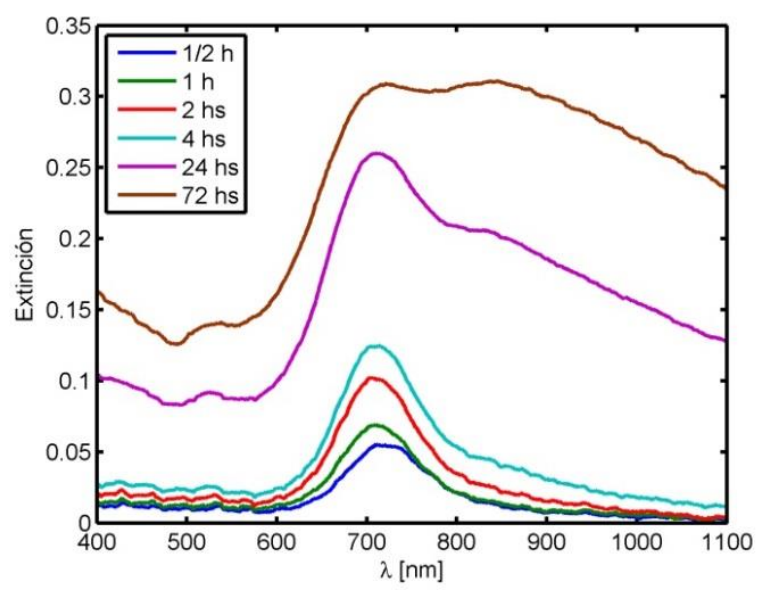

Figura 3: Espectros de extinción de los portaobjetos con distintos tiempos de inmersión en el coloide. Se observa el crecimiento del pico correspondiente al plasmón longitudinal de los NBOs a medida que van recubriendo la superficie del sustrato. Además, a partir de un día de inmersión, se observa un ensanchamiento del pico debido a la presencia de interacciones entre las nanopartículas adsorbidas.

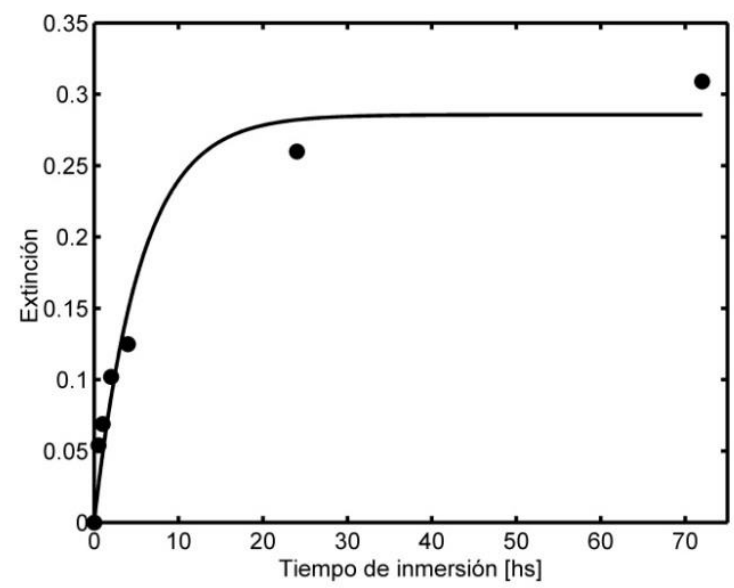

Figura 4: Amplitud de los picos de extinción correspondientes a la resonancia longitudinal de los NBOs, en función del tiempo de inmersión de los portaobjetos en la solución coloidal. Se observa un comportamiento asintótico del recubrimiento hacia una densidad máxima de saturación (dada por una repulsión electrostática entre las nanopartículas) y un tiempo propio de este proceso, de entre 5 y 6 horas.

Se observa además en los espectros de los sustratos recubiertos un leve corrimiento de los picos de extinción hacia longitudes de onda menores debido al cambio de entorno químico, pues la solución coloidal es acuosa y cuando los NBOs están adsorbidos al vidrio, rodeados de aire, pasan a estar rodeados de un índice de refracción efectivo menor.

En los espectros de la Figura 3 se observa también que a partir de 24 horas de inmersión, el pico del plasmón longitudinal se ensancha hacia regiones del infrarrojo. Las interacciones entre nanopartículas metálicas producen un corrimiento al rojo del plasmón, y este corrimiento es mayor cuanto más intenso es el acople $[29, \underline{30}]$. Estas interacciones que muestran los espectros se pueden deber a cierta movilidad de los NBOs sobre las moléculas de PSS mientras el sustrato está sumergido en la solución coloidal, formando en principio pequeños agregados como dímeros y trímeros, y a medida que pasa el tiempo se van formando agregados de mayor número de partículas.

La capa de surfactante alrededor de los NBOs actúa como una barrera evitando que las nanopartículas se toquen, situándolas a una cierta distancia inter-partícula dada por el espesor de la bicapa de CTAB. Esta distancia promedio entre los NBOs agregados genera un acople plasmónico cuya resonancia está corrida al rojo respecto del caso aislado, tal como se observa en los espectros correspondientes a 24 horas y 72 horas de inmersión de la Figura 3. La aparición de este ensanchamiento del espectro marca el comienzo del proceso de agregación de los NBOs. 
En estos dos últimos espectros se puede observar también la aparición de un pequeño pico apenas por encima de los $500 \mathrm{~nm}$, correspondiente al plasmón transversal de los NBOs. Su menor amplitud respecto del pico longitudinal se debe a que esta resonancia posee una sección eficaz de absorción y dispersión significativamente menor.

La imagen SEM de la Figura 5 muestra los NBOs que recubren un sustrato de vidrio luego de 24 horas de inmersión en la solución coloidal. Pueden verse nanopartículas aisladas y una cantidad importante de agregados, formando dímeros o grupos de mayor número de nanopartículas. Los NBOs que forman parte de estos agregados se acoplan plasmónicamente y generan la mencionada extensión de la resonancia hacia regiones del infrarrojo.

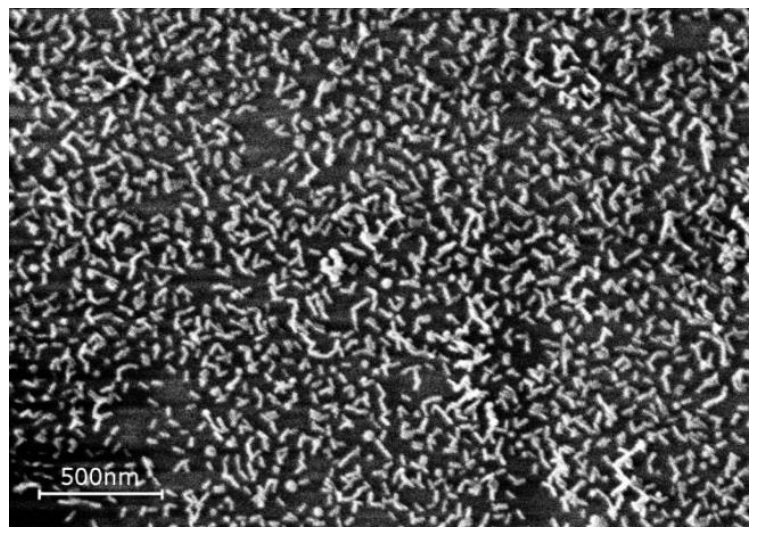

Figura 5: Imagen SEM de la superficie de un portaobjetos recubierta de NBOs por el método descripto en este trabajo, para un tiempo de inmersión de 24 horas. Se observa una alta densidad superficial de NBOs, y la aparición de agregados de dos o más NBOs que poseen resonancias corridas al rojo, produciendo un ensanchamiento del plasmón longitudinal.

\section{CONCLUSIONES}

Se sintetizaron con éxito NBOs con muy baja dispersión de tamaño y una alta eficiencia. Se modificaron químicamente sustratos de vidrio para recubrirlos con los NBOs sintetizados por inmersión en coloide. Se estudió este proceso por espectroscopía a través del crecimiento y evolución de las resonancias plasmónicas y se obtuvo control en la densidad superficial de NBOs. Se observó una primera etapa de recubrimiento homogéneo y luego una etapa de agregación caracterizada por interacciones plasmónicas entre los NBOs.

\section{AGRADECIMIENTOS}

Este trabajo fue realizado gracias al financiamiento del subsidio PID-UTI1624 de la Universidad Tecnológica Nacional.

\section{BIBLIOGRAFÍA}

[1] KABASHIN, A.V., EVANS, P., PASTKOVSKY, S., et al., "Plasmonic nanorod metamaterials for biosensing", Nature Materials, v. 8, pp. 867-871, Oct. 2009.

[2] LIU, N., TANG, M.L., HENTSCHEL, M., et al., "Nanoantenna-enhanced gas sensing in a single tailored nanofocus”, Nature Materials, v. 10, pp. 631-636, May. 2011.

[3] NOGINOV, M.A., ZHU, G., BELGRAVE, A.M., et al., "Demonstration of a spaser-based nanolaser", Nature, v. 460, pp. 1110-1112, Ago. 2009.

[4] KINKHABWALA, A., YU, Z., FAN, S., et al., "Large single-molecule fluorescence enhancements produced by a bowtie nanoantenna", Nature Photonics, v. 3, pp. 654-657, Oct. 2009.

[5] YUAN, H., KHATUA, S., ZIJLSTRA, P., et al., "Thousand-fold Enhancement of Single-Molecule Fluorescence Near a Single Gold Nanorod”, Angewandte Chemie International Edition, v. 52, n. 4, pp. 1217-1221, Ene. 2013.

[6] HUANG, X., JAIN, P.K., EL-SAYED, I.H., et al., "Plasmonic photothermal therapy (PPTT) using gold nanoparticles", Lasers in Medical Science, v. 23, n. 3, pp. 217-228, Jul. 2008.

[7] BARDHAN, R., LAL, S., JOSHI, A., et al., "Theranostic Nanoshells: From Probe Design to Imaging and Treatment of Cancer", Accounts of Chemical Research, v. 44, n. 10, pp. 936-946, May. 2011.

[8] BRAGAS, A.V., MARTÍNEZ, O.E., "Field-enhanced scanning optical microscope”, Optics Letters, v. 25, 
n. 9, pp. 631-633, May. 2000.

[9] SCARPETTINI, A.F., PELLEGRI, N., BRAGAS, A.V., "Optical imaging with subnanometric vertical resolution using nanoparticle-based plasmonic probes”, Optics Communications, v. 282, n. 5, pp. 1032-1035, Mar. 2009.

[10] ATWATER, H.A., POLMAN, A., "Plasmonics for improved photovoltaic devices", Nature Materials, v. 9, pp. 205-213, Feb. 2010.

[11] NUSZ, G.J., CURRY, A.C., MARINAKOS, S.M., et al., "Rational Selection of Gold Nanorod Geometry for Label-Free Plasmonic Biosensors", ACS Nano, v. 3, n. 4, pp. 795-806, Mar. 2009.

[12] KREIBIG, U., VOLLMER, M., Optical Properties of Metal Clusters, 1 ed., Berlin, Springer-Verlag, 1995.

[13] PERASSI, E.M., SCARPETTINI, A.F., MASIP, M.E., et al., "Understanding the Behavior of New Plasmonic Probes with Sub-Nanometric Resolution in Field Enhanced Scanning Optical Microscopy", Journal of Physical Chemistry C, v. 115, n. 21, pp. 10455-10461, May. 2011.

[14] SAU, T.K., MURPHY, C.J., "Self-Assembly Patterns Formed upon Solvent Evaporation of Aqueous Cetyltrimethylammonium Bromide-Coated Gold Nanoparticles of Various Shapes”, Langmuir, v. 21, n. 7, pp. 2923-2929, Mar. 2005.

[15] GUERRERO-MARTÍNEZ, A., PÉREZ-JUSTE, J., CARBÓ-ARGIBAY, E., et al., "Gemini-SurfactantDirected Self-Assembly of Monodisperse Gold Nanorods into Standing Superlattices", Angewandte Chemie International Edition, v. 48, n. 50, pp. 9484-9488, Dic. 2009.

[16] NIIDOME, Y., TAKAHASHI, H., URAKAWA, S., et al., "Immobilization of Gold Nanorods on the Glass Substrate by the Electrostatic Interactions for Localized Plasmon Sensing", Chemistry Letters, v. 33, n. 4, pp. 454-455, Mar. 2004.

[17] FERHAN, A.R., GUO, L., KIM, D.H., "Influence of Ionic Strength and Surfactant Concentration on Electrostatic Surfacial Assembly of Cetyltrimethylammonium Bromide-Capped Gold Nanorods on Fully Immersed Glass", Langmuir, v. 26, n. 14, pp. 12433-12442, Jun. 2010.

[18] GOLE, A., ORENDORFF, C.J., MURPHY, C.J., "Immobilization of Gold Nanorods onto AcidTerminated Self-Assembled Monolayers via Electrostatic Interactions”, Langmuir, v. 20, n. 17, pp. 71177122, Jul. 2004.

[19] GOLE, A., MURPHY, C.J., "Polyelectrolyte-Coated Gold Nanorods: Synthesis, Characterization and Immobilization”, Chemistry of Materials, v. 17, n. 6, pp. 1325-1330, Feb. 2005.

[20] GOLE, A., MURPHY, C.J., "Biotin-Streptavidin-Induced Aggregation of Gold Nanorods: Tuning Rod-Rod Orientation”, Langmuir, v. 21, n. 23, pp. 10756-10762, Oct. 2005.

[21] HONDA, K., KAWAZUMI, H., NAKASHIMA, N., et al., "Label-free Biosensor Using Polyionmodified Gold Nanorods Adsorbed on a Glass Substrate”, Chemistry Letters, v. 39, n. 9, pp. 992-993, Ago. 2010.

[22] NIIDOME, Y., NAKAMURA, Y., HONDA, K., et al., "Spectral dependence of gold nanorods on the optical properties of substrates and adsorption of polypeptides", Journal of Photochemistry and Photobiology A, v. 221, n. 2-3, pp. 204-208, Jun. 2011.

[23] WANG, Y., TANG, L., "Chemisorption assembly of Au nanorods on mercaptosilanized glass substrate for label-free nanoplasmon biochip”, Analytica Chimica Acta, v. 796, pp. 122-129, Sep. 2013.

[24] CAO, J., GALBRAITH, E.K., SUN, T., et al., "Effective surface modification of gold nanorods for localized surface plasmon resonance-based biosensors", Sensors and Actuators B, v. 169, pp. 360-367, Jul. 2012.

[25] JANA, N.R., GEARHEART, L., MURPHY, C.J., "Wet Chemical Synthesis of High Aspect Ratio Cylindrical Gold Nanorods”, Journal of Physical Chemistry B, v. 105, n. 19, pp. 4065-4067, Abr. 2001.

[26] NIKOOBAKHT, B., EL-SAYED, M.A., "Preparation and Growth Mechanism of Gold Nanorods (NRs) Using Seed-Mediated Growth Method", Chemistry of Materials, v. 15, n. 10, pp. 1957-1962, Abr. 2003.

[27] YE, X., JIN, L., CAGLAYAN, H., et al., "Improved Size-Tunable Synthesis of Monodisperse Gold Nanorods through the Use of Aromatic Additives", ACS Nano, v. 6, n. 3, pp. 2804-2817, Feb. 2012.

[28] SCARPETTINI, A.F., BRAGAS, A.V., "Coverage and Aggregation of Gold Nanoparticles on Silanized Glasses”, Langmuir, v. 26, n. 20, pp. 15948-15953, Sep. 2010.

[29] KHLEBTSOV, B., MELNIKOV, A., ZHAROV, V., et al., "Absorption and scattering of light by a dimer of metal nanospheres: comparison of dipole and multipole approaches", Nanotechnology, v. 17, n. 5, pp. 1437-1445, Feb. 2006. 
GUTIÉRREZ, M.V.; PAREDES, M.Y.; SCARPETTINI, A.F. revista Matéria, v. 20, n. 3, pp. 731- 738, 2015.

[30] ROMERO, I., AIZPURUA, J., BRYANT, G.W., et al., "Plasmons in nearly touching metallic nanoparticles: singular response in the limit of touching dimers", Optics Express, v. 14, n. 21, pp. 9988-9999, Oct. 2006. 